\title{
Study of high sensitive c-reactive protein in preeclampsia
}

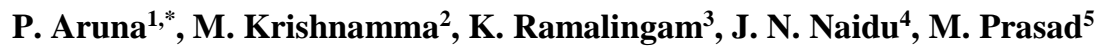 \\ ${ }^{1}$ Assistant Professor, ${ }^{2}$ Professor, ${ }^{3}$ Associate Professor, ${ }^{4}$ Prfoessor \& HOD, ${ }^{5}$ Tutor, Dept. of Biochemistry, ${ }^{1}$ ACSR Governemnt \\ Medical College, Nellore, Andhra Pradesh, ${ }^{2,3,4,5}$ Narayana Medical College and Hospital, Chinthareddypalem, Nellore, Andhra \\ Pradesh, India
}

*Corresponding Author:

Email: draruna77@gmail.com

Received: $27^{\text {th }}$ October, 2017

Accepted: $05^{\text {th }}$ March, 2018

\begin{abstract}
Introduction: Preeclampsia is a serious complication of the second half of pregnancy and is an important cause of maternal and perinatal morbidity and mortality. Measurement of circulatory inflammatory markers of endothelial cell dysfunction and inflammation may provide an alternative method of detecting women at high risk of delivery. Women with a history of preeclampsia have an increased succeptibiliy to lipoprotein oxidation when compared to women who had normal pregnancy. Aims and objectives: The present study was designed to study hsCRP in association with blood pressure, proteinuria and lipid profile as markers of inflammation and endothelial dysfunction in preeclampsia.

Materials and Methods: The present study was carried out for one year on diagnosed patients of Preeclampsia attending the outpatient and inpatient departments of obstetrics and Gynecology department of Narayana Medical College and Hospital, Nellore. The study was undertaken on 50 Preeclampsia cases and 50 normal pregnant women as controls. Results: In our study hs-CRP levels were significantly elevated in cases when compared to control subjects. Total cholesterol, triacylglycerol and LDL values were elevated in total cases when compared to controls. However the decrease in HDL cholesterol was not significant when compared to controls.

Conclusion: Elevated levels of LDL undergo oxidation to form oxidized LDL, which is involved in inflammation and free radical generation. Increased inflammation leads to increased levels of hsCRP, which is further associated with dyslipidemia, an important cardiovascular risk factor in preeclampsia.
\end{abstract}

Keywords: Preeclampsia, hsCRP, Systolic Blood pressure, Dyslipidemia, urine protein creatinine ratio (P/C ratio), Pregnancy induced hypertension.

\section{Introduction}

Preeclampsia develops in $4-5 \%$ of human pregnancies and is characterised by hypertension, dyslipidemia and increased systemic inflammatory response. ${ }^{1}$ Pregnancy Induced Hypertension (PIH) is a serious complication of thesecond half of pregnancy with urinary loss of proteins, oedema and activation of hemostatic mechanisms. ${ }^{2}$

Preeclampsia is characterized by an increased BP equal to or above $140 / 90 \mathrm{mmHg}$ in the presence of proteinuria that develops after 20 weeks of gestational age. PE results in eclampsia if convulsion develops or manifests as hemolysis, elevated liver enzymes and low platelet count (HELLP) syndrome. Severe complication like cerebral hemorrhage, renal failure, lung edema and liver hemorrhage are associated with eclampsia and HELLP syndrome. ${ }^{3}$

Endothelial cell dysfunction and inflammation have an important role in the pathophysiological mechanism of preeclampsia. There is Increasing clinical and biochemical evidence which suggests that disturbance of normal endothelial cell function may be a primary cause in the pathogenesis of $\mathrm{PE} .{ }^{4} \mathrm{C}$-reactive protein (CRP) is one of the major acute-phase reactant in humans. It is an important first-line host defence molecule as it activates the complement system and mediates the phagocytic clearance of pathogens and damaged cells. ${ }^{5.6}$

The term high sensitivity CRP (hsCRP) refers to the lower detection limit of the assay procedures being used and is otherwise similar to routine CRP in structure and function. It has been suggested that hsCRP has provided better sensitivity in establishing inflammation than levels of CRP gives an idea about ongoing inflammation and tissue damage very accurately compared to other laboratory parameters of the acute-phase response. ${ }^{7}$ The hs-CRP is a sensitive marker of tissue damage and inflammation.It is useful in differentiating acute inflammation as well as assessment of severity of inflammation. ${ }^{8}$

Mean plasma triglyceride and free fatty acid concentrations undergo near doubling in women with preeclampsia relative to normal pregnancy. ${ }^{9}$ The hypertriglyceridemia of preeclampsia is also accompanied by increased prevalence of smaller, denser LDL particles and decreased HDL cholesterol. Triacylglycerol levels are a major determinant of cholesterol ester transfer protein (CETP) activity. ${ }^{10}$ Therefore, in the presence of increased triacylglycerolrich lipoproteins, CETP activity is increased so that all circulating lipoproteins become enriched in triacylglycerol, in particular HDL and LDL particles. ${ }^{11}$

LDL particles are triacylglycerol enriched due to the hyper triglyceridemia and increased CETP activity. 
They are also converted by the triglyceride lipase activity of hepatic lipase into smaller and denser particles. Large buoyant LDL is cleared rapidly by the LDL receptor pathway and small dense LDL is removed more slowly. ${ }^{12}$ Triacylglycerol-rich HDL particles are converted by the triglyceride lipase activity of hepatic lipase into smaller particles which are better substrates for catabolic pathways. ${ }^{11}$

One of the reasons for this dyslipidemia is due to hormonal imbalance in preeclampsia. Preeclampsia is a state of hypoestrogenemia. There is impairment in the formation of Dehydroepiandrosterone (DHEA) by fetal adrenal glands due to decreased uteroplacental blood flow there by leading to decreased uptake of lipids by the fetus. ${ }^{13}$ DHEA is the important source of estrogen in pregnancy. The decrease in utero-placental blood flow leads to relative stasis of maternal blood in the intervillous space which leads to redistribution of steroids formed in syncytium back to the fetus instead of entering into maternal circulation which also results in a state of hypoestrogenemia. ${ }^{13}$

Oxidative stress has been implicated in the pathogenesis of several complication of human pregnancy including preeclampsia. In preeclampsia dyslipidemia pattern of increased concentration of triglyceride, cholesterol and LDL and decreased concentration of HDL have been noticed Preeclampsia is associated with elevated lipid peroxidation and reduced antioxidant status. Preeclampsia is an endothelial disease with a major involvement of lipid mediated oxidative damage. ${ }^{14}$

The present study was designed to establish an association among blood pressure, proteinuria, hsCRP and lipid profile as markers of inflammation and endothelial dysfunction in pregnancy induced hypertension.

\section{Materials and Methods}

The present study was carried out for one year on diagnosed patients of preeclampsia attending the outpatient and inpatient departments of Obstetrics and Gynecology department of Narayana Medical College and Hospital, Nellore. The study was undertaken on 50 preeclampsia cases and 50 normal pregnant women.

Inclusion criteria: 50 normal healthy pregnant women of $>20$ weeks of gestational age were selected as controls. 50 diagnosed preeclampsia cases $>20$ weeks of gestational age and with urine protein creatinine ratio ( $\mathrm{P} / \mathrm{C}$ ratio) more than 0.3 were selected as cases for the study.

Exclusion criteria: Pregnant women with diabetes mellitus, anemia and other chronic illnesses were excluded from the study. Women with the following conditions were also excluded: a known kidney disease, heavy exercise (more than 1 hour of vigorous exercise on the day of urine collection), bacteriuria, bed rest longer than 24 hours, and gestational diabetes mellitus.
In addition, women who delivered their babies during the urine collection day were excluded.

$6 \mathrm{ml}$ venous blood was collected from the cases and controls under aseptic precautions and it was transferred to the Plain tube and serum was separated by centrifugation and stored at $-20^{\circ} \mathrm{C}$ to measure the analytes. Haemolysed and lipemic samples were avoided. $5 \mathrm{ml}$ of the collected urine sample was centrifuged, supernatant separated and stored at $-20^{\circ} \mathrm{C}$ to measure the analytes.

hsCRP was determined by turbidometric immunoassay using commercially available (Euro diagnostics) kit method.Total cholesterol, triacylglycerol, HDL, LDL, urine microprotein and urine creatinine were estimated by commercially available kits in a semi auto analyser.

The mean and standard deviation were calculated for all the Biochemical parameters. The significance between the groups were determined using Student $\mathbf{t}$ - test for Equality of means. The p-value of < $\mathbf{0 . 0 5}$ was considered significant.

\section{Results}

In the present study the mean \pm SD of SBP in controls $(n=50)$ and pre-eclamptic cases $(n=50)$ were $110.8 \pm \quad 8.0$ and $159.2 \pm 16.87 \mathrm{~mm}$ of $\mathrm{Hg}$ respectively.This increase was statistically significant $(\mathrm{p}<0.0001)$ as shown in Table 1$)$.

The mean \pm SD of DBP in controls $(n=50)$ and preeclamptic cases $(\mathrm{n}=50)$ were $73.6 \pm 5.6$ and $103.56 \pm$ $8.95 \mathrm{~mm}$ of $\mathrm{Hg}$ respectively.This increase was statistically significant $(\mathrm{p}<0.0001)$ as shown in (Table 1, Fig. 1). The mean \pm SD of urine $\mathrm{P} / \mathrm{C}$ ratio in controls $(\mathrm{n}=50)$ and pre-eclamptic cases $(\mathrm{n}=50)$ were $0.13 \pm$ 0.02 and $0.64 \pm 0.26$ respectively. This increase was statistically significant $(\mathrm{p}<0.0001)$ as shown in Table $1)$.

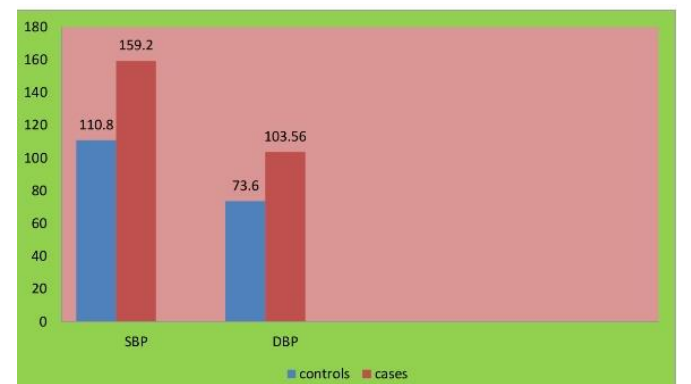

Fig. 1: Comparison of mean SBP and DBP levels in controls and PE cases 


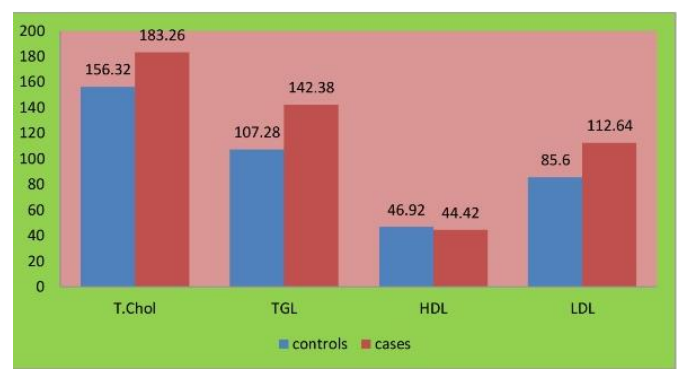

Fig. 2: Comparison of mean total cholesterol, TGI, HDL-C and LDL-C levels in cases and controls

In our present study total cholesterol, triacylglycerol and LDL values were elevated in total cases when compared to controls (P-value $<0.05$, Table 1, Fig. 2). The decrease in HDL cholesterol was not significant when compared to controls (Table 1). The mean \pm SD of hsCRP in controls $(\mathrm{n}=50)$ and pre-eclamptic cases $(\mathrm{n}=50)$ were $1.71 \pm 0.85$ and $8.07 \pm 2.09 \mathrm{mg} / \mathrm{L}$ respectively. hs-CRP levels in our study were significantly elevated in cases when compared to control subjects(Table 1, P-value<0.0001, Fig. 3).

Correlation analysis of study variables was done among cases. $\mathrm{P}$ value less than 0.05 was considered significant. In our study hsCRP correlated positively with SBP, DBP and $\mathrm{P} / \mathrm{C}$ ratio. SBP levels in cases correlated positively with $\mathrm{P} / \mathrm{C}$ ratio (Figure No.4), Total cholesterol, LDL and hsCRP.

$\mathrm{P} / \mathrm{C}$ ratio in our study correlated positively with Total cholesterol, triacyl glycerol, LDL and hscRP. (Pvalue $<0.05$, Table 2 ).

Table 1: Biochemical Parameters in Cases and Controls

\begin{tabular}{|c|l|c|c|c|c|c|c|}
\hline \multirow{2}{*}{ S. No. } & \multirow{2}{*}{ Parameter } & \multicolumn{2}{|c|}{ Controls } & \multicolumn{2}{c|}{ Cases } & \multirow{2}{*}{ P value } & \multirow{2}{*}{ value } \\
\cline { 3 - 7 } & & Mean & S.D. & Mean & S.D. & & \\
\hline 1. & SBP $\mathrm{mmHg}$ & 110.8 & 8.0 & 159.2 & 16.87 & $<0.0001$ & 18.33 \\
\hline 2 & DBP $\mathrm{mmHg}$ & 73.6 & 5.62 & 103.56 & 8.95 & $<0.0001$ & 20.04 \\
\hline 3 & P/C ratio & 0.13 & 0.02 & 0.64 & 0.26 & $<0.0001$ & 13.82 \\
\hline 4 & $\begin{array}{l}\text { T.Cholesterol } \\
\text { mg/dl }\end{array}$ & 156.32 & 32.04 & 183.26 & 40.39 & 0.0004 & 3.695 \\
\hline 5 & TGL mg/dl & 107.28 & 37.2 & 142.38 & 32.49 & $<0.0001$ & 5.02 \\
\hline 6 & HDL-C mg/dl & 46.92 & 12.2 & 44.42 & 10.39 & 0.27 & 1.10 \\
\hline 7 & LDL-C mg/dl & 85.6 & 18.86 & 112.64 & 27.24 & $<0.0001$ & 5.77 \\
\hline 8 & hsCRP mg/L & 1.71 & 0.85 & 8.07 & 2.09 & $<0.0001$ & 19.93 \\
\hline
\end{tabular}

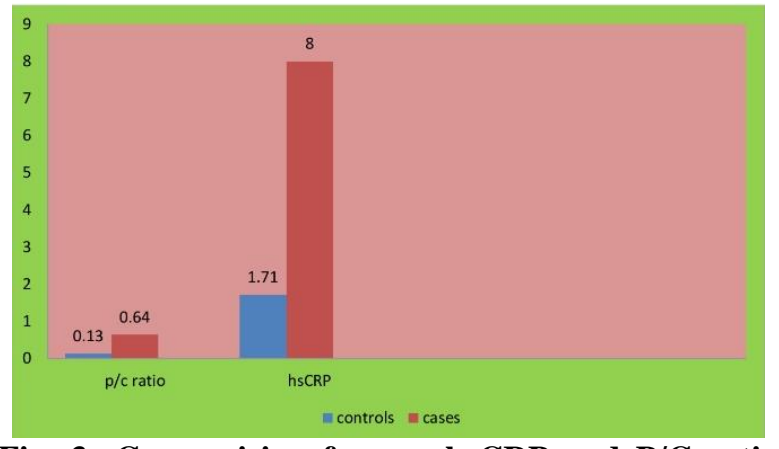

Fig. 3: Comparisin of mean hsCRP and $\mathrm{P} / \mathrm{C}$ ratio levels in cases and controls

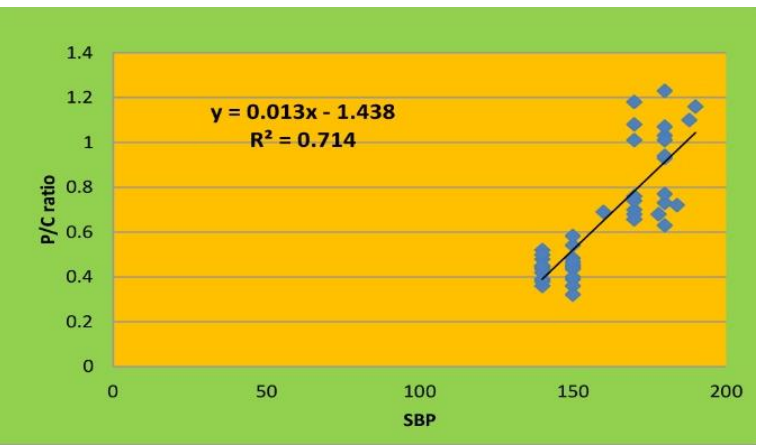

Fig. 4: Scatter diagram showing correlation between SBP Vs. P/C ratio in cases

Table 2: Correlation analysis of study variables

\begin{tabular}{|l|c|c|c|c|c|c|c|c|}
\hline hsCRP Vs & \multicolumn{2}{|l|}{ SBP Vs } & & & $\begin{array}{l}\text { P/C } \\
\text { ratio Vs }\end{array}$ & \multicolumn{2}{l|}{} \\
\hline & r-value & p-value & & r-value & p-value & & r-value & p-value \\
\hline SBP & 0.55 & $<0.05$ & P/C ratio & 0.84 & $<0.05$ & T. Chol & 0.32 & $<0.05$ \\
\hline DBP & 0.62 & $<0.05$ & T. Chol & 0.36 & $<0.05$ & TAG & 0.34 & $<0.05$ \\
\hline P/C ratio & 0.49 & $<0.05$ & LDL & 0.35 & $<0.05$ & LDL & 0.3 & $<0.05$ \\
\hline & & & hsCRP & 0.55 & $<0.05$ & hsCRP & 0.49 & $<0.05$ \\
\hline
\end{tabular}

\section{Discussion}

The present study was conducted on fifty normal healthy pregnant women as controls and fifty preeclamptic women as cases. Pregnancy Induced Hypertension (PIH) is a pregnancy specific disorder that adversely affects the mother by vascular dysfunction and the fetus by intrauterine growth 
retardation. ${ }^{9}$ The association of alteration of serum lipid profile with PIH is well documented. An abnormal lipid profile is known to be strongly associated with atherosclerotic cardiovascular diseases. In our present study total cholesterol, triacylglycerol and LDL values were elevated in total cases when compared to controls (P-value $<0.05$, table 1). The decrease in HDL cholesterol was not significant when compared to controls (Table 1). This was in accordance with the study done by J. T. Gohil etal, ${ }^{15}$ I. Cuneyt Evruke etal, ${ }^{16}$ Pradnya Phalak et al, ${ }^{17}$ Jayanta De. ${ }^{18}$

Endothelial cell dysfunction is considered to have a crucial role in the pathophysiological mechanism of preeclampsia and is accompanied by elevated levels of inflammatory markers. Indeed, such marker levels have been shown to be much higher in women with PE compared to normal pregnant ladies. ${ }^{4}$ The hs-CRP is a sensitive marker of tissue damage and inflammation. It is useful in differentiating acute inflammation as well as assessment of severity of inflammation. ${ }^{8}$

In our present study hs-CRP was significantly elevated in cases when compared to control subjects (Table 1, P-value<0.0001). This was in accordance with the study conducted by Hossein Ayatollahi et al, ${ }^{4}$ Teran etal, ${ }^{19}$ Batashki I etal. ${ }^{20}$ Hossein Ayatollahi etal determined the serum concentration of hsCRP in normal pregnancy and preeclampsia and concluded that hsCRP levels are elevated in severe preeclampsia compared with mild preclampsia and normal pregnancy.

Nanda $\mathrm{K}$ et $\mathrm{al}^{21}$ showed a significant positive correlation between hsCRP and BMI as well as lipid parameters. In a study conducted by T. Sarala b Devi et $\mathrm{al}^{22}$ the mean Triglycerides and VLDL cholesterol levels were significantly increased in cases as compared to controls and they concluded that hsCRP could be used as a predictive factor for pre-eclampsia. Human CRP being a calcium dependent ligand binding protein with highest affinity to phosphocholine residues in plasma lipoproteins; formed the basis for their observations.

In our study hsCRP correlated positively with SBP, $\mathrm{DBP}$ and $\mathrm{P} / \mathrm{C}$ ratio. There was positive correlation of $\mathrm{P} / \mathrm{C}$ ratio with lipid parameters indicating that dyslipidemia was associated with poteinuria.

We could also establish a positive correlation of SBP with P?C ratio in our study. (Table 2). Rani Sauriasari et al in their study demonstrated a significant association among the hs-CRP, a high eGFR and high urinary protein in current smokers. ${ }^{23}$

There is an association among transvasular albumin leakage, proteinuria and vascular permeability in endothelial dysfunction. Increased CRP being a marker of endothelial dysfunction may directly alter renal vascular permeability, and thus be causally involved in the hyperfiltration of remnant glomeruli, resulting in the development of proteinuria. ${ }^{23}$
Albuminuria serves as a surrogate marker of increased glomerular capillary pressure and a predictor of progressive renal injury. There might be lower eGFR when there is proteinuria and produces significant renal injury in association with hypertension, which further leads to cardiovascular and renal complications. ${ }^{24}$

\section{Conclusion}

Preeclampsia is one of the leading causes of maternal mortality and morbidity. In preeclampsia there is an exaggeration of systemic inflammatory response that might induce reactive oxygen species which further induces endothelial dysfunction. This leads to clinical symptoms of hypertension and proteinuria in preeclampsia. The hs-CRP is sensitive marker of tissue damage and inflammation. The present study showed that hsCRP was elevated in preeclampsia. Dyslipidemia was associated with preeclampsia, which acts as a precursor for sub clinical atherosclerotic progression in PIH. In addition, hypertension in pregnancy is considered as a marker for the later development of cardiovascular and renal morbidity.

\section{References}

1. Farzadnia, Mehdi, Hossein Ayatollahi, Maliheh Hasanzade, and Hamid Reza Rahimi. "A Comparative Study of Serum Level of Vascular Cell Adhesion Molecule-1 (sVCAM-1), Intercellular Adhesion Molecule-1(ICAM1) and High Sensitive C - Reactive Protein (Hs-CRP) in Normal and Pre-Eclamptic Pregnancies.” Iranian Journal of Basic Medical Sciences 16, no. 5 (May 2013):689-93.

2. Roberts, J. M., and D. W. Cooper. "Pathogenesis and Genetics of Pre-Eclampsia." Lancet (London, England) 357, no. 9249 (January 6, 2001):53-6.

3. Kumru, Selahattin, Ahmet Godekmerdan, Selim Kutlu, and Zeynep Ozcan. "Correlation of Maternal Serum High-Sensitive C-Reactive Protein Levels with Biochemical and Clinical Parameters in Preeclampsia." European Journal of Obstetrics, Gynecology, and Reproductive Biology 124, no. 2 (February 1, 2006):1647. https://doi.org/10.1016/j.ejogrb.2005.05.007

4. Ayatollahi, Hossein, Maliheh Hasanzade, Mahdi Farzadnia, Mahdi Khabbaz Khoob, and Atefeh Rahmanian. "Serum Level of High Sensitive C-Reactive Protein in Normal and Preeclamptic Pregnancies." Iranian Journal of Pathology 2, no. 3 (June 1, 2007):1004.

5. Mortensen, R. F. "C-Reactive Protein, Inflammation, and Innate Immunity.” Immunologic Research 24, no. 2 (2001): 163-76. https://doi.org/10.1385/IR:24:2:163

6. Du Clos, T. W. "Function of C-Reactive Protein." Annals of Medicine 32, no. 4 (May 2000):274-8.

7. Dhok, Archana J., Sangeeta Daf, Kanchan Mohod, and Satish Kumar. "'Role of Early Second Trimester High Sensitivity C-Reactive Protein for Prediction of Adverse Pregnancy Outcome'.," 2011.

8. Anil Bargale, JayashreeV.Ganu, Dhirajj.Trivedi, Nitin Nagane, Rakesh Mudaraddi, Aparna Sagare "Serum hsCRP \& uric acid as indicator of severity in preeclampsia". International Journal of Pharma and Bio Sciences 2, no3(Jul-Sept 2011)B340-5. 
9. Hubel, Carl A. "Oxidative Stress in the Pathogenesis of Preeclampsia." Proceedings of the Society for Experimental Biology and Medicine 222, no. 3 (1999):222-35.

10. Mann, C. J., F. T. Yen, A. M. Grant, and B. E. Bihain. "Mechanism of Plasma Cholesteryl Ester Transfer in Hypertriglyceridemia." The Journal of Clinical Investigation 88, no. 6 (December 1991): 2059-66. https://doi.org/10.1172/JCI115535

11. Patsch, J R, S Prasad, A M Gotto, and G BengtssonOlivecrona. "Postprandial Lipemia. A Key for the Conversion of High Density lipoprotein2 into High Density lipoprotein 3 by Hepatic Lipase." Journal of Clinical Investigation 74, no. 6 (December 1984):201723.

12. Packard, C. J., and J. Shepherd. "Lipoprotein Heterogeneity and Apolipoprotein B Metabolism." Arteriosclerosis, Thrombosis, and Vascular Biology 17, no. 12 (December 1997):3542-56.

13. Pushparaj, Josephine Latha, and Ganesan Subramanyam. "Dyslipidemia in Pre-Eclampsia-Risk Factor for Future Maternal Cardiovascular Diseases." Journal of Evolution of Medical and Dental Sciences 1, no. 4 (2012):487-95.

14. Meera, K. S., S. Maitra, and R. Hemalatha. "Increased Level of Lipid Peroxidation in Preeclamptic Pregnancy; a Rela-Tionship with Paraoxanase 1(PON1) Activity." Biomedical Research, 2010. http://www.biomedres.info/abstract/increased-level-oflipid-peroxidation-in-preeclamptic-pregnancy-arelationship-with-paraoxanase-1pon1-activity-1344.html

15. Gohil, J. T., P. K. Patel, and Priyanka Gupta. "Estimation of Lipid Profile in Subjects of Preeclampsia." Journal of Obstetrics and Gynaecology of India 61, no. 4 (August 2011): 399-403. https://doi.org/10.1007/s13224-0110057-0

16. Evrüke, I. Cüneyt, S. Cansun Demir, Ibrahim F. Urünsak, F. Tuncay Ozgünen, and Oktay Kadayifçi. "Comparison of Lipid Profiles in Normal and Hypertensive Pregnant Women." Annals of Saudi Medicine 24, no. 5 (October 2004):382-5.

17. Phalak, Pradnya, and Mona Tilak. "Study of Lipid Profile in Pre-Eclampsia." Indian Journal of Basic \& Applied Medical Research 5, no. 2 (2012):405-9.

18. De, Jayanta, Ananda Mukhopadhyay, and Pradip Kumar Saha. "Study of Serum Lipid Profile in Pregnancy Induced Hypertension." Indian Journal of Clinical Biochemistry 21, no. 2 (2006):165-8.

19. 19 Teran, E., C. Escudero, W. Moya, M. Flores, P. Vallance, and P. Lopez-Jaramillo. "Elevated C-Reactive Protein and pro-Inflammatory Cytokines in Andean Women with Pre-Eclampsia." International Journal of Gynaecology and Obstetrics: The Official Organ of the International Federation of Gynaecology and Obstetrics 75, no. 3 (December 2001):243-9.

20. Batashki I, Milchev N, Topalovska D. et al. " $\mathrm{C}$ - reactive protein in women with preeclampsia." Akush Ginecol (soffia) 45,no 1.(2006),47-50.

21. 21 Nanda K, Sadanand G. Muralidhara Krishna C S, K L Mahadevappa "C-Reactive Protein as a Predictive Factor of Preeclampsia." Int J Biol Med Res (Jan 2012)3(1),1307-10

https://www.biomedscidirect.com/509/c_reactive_protein _as_a_predictive_factor_of_preeclampsia/articlescategori es22

22. 22 Devi, T. Sarala, Penugonda Anveetha, and Sujatha Pasula. "HIGH SENSITIVE C-REACTIVE PROTEIN IN PRE-ECLAMPSIA" IJPBS 3, no 3 (. Jul-Sep 2013).27-32.
23. Sauriasari, Rani, Noriko Sakano, Da-Hong Wang, Jiro Takaki, Kei Takemoto, BingLing Wang, Hitoshi Sugiyama, et al. "C-Reactive Protein Is Associated with Cigarette Smoking-Induced Hyperfiltration and Proteinuria in an Apparently Healthy Population." Hypertension Research 33, no. 11 (November 2010): 1129. https://doi.org/10.1038/hr.2010.154n

24. Yan, Ping, Xiangzhu Zhu, Haiming Li, Martha J. Shrubsole, Haiming Shi, Ming-zhi Zhang, Raymond C. Harris, Chuan-Ming Hao, and Qi Dai. "Association of High Blood Pressure with Renal Insufficiency: Role of Albuminuria, from NHANES, 1999-2006." PLOS ONE 7, no. 7 (July 3, 2012). https://doi.org/10.1371/journal.pone.0037837. 